Epidemiology and Infection

cambridge.org/hyg

\section{Original Paper}

Cite this article: Endo A, Funk S, Kucharski AJ (2020). Bias correction methods for testnegative designs in the presence of misclassification. Epidemiology and Infection 148, e216, 1-12. https://doi.org/10.1017/ S0950268820002058

Received: 26 April 2020

Revised: 24 July 2020

Accepted: 28 August 2020

\section{Key words:}

Misclassification bias; sensitivity; specificity; test-negative design; vaccine effectiveness

\begin{abstract}
Abbreviations:
VE: vaccine effectiveness; TND: test-negative design; TD: target disease; ND: non-target disease; PCR: polymerase chain reaction; MLE: maximum likelihood estimate; MO: multiple overimputation; EM: expectation-maximisation
\end{abstract}

\section{Author for correspondence:}

A. Endo,

E-mail: akira.endo@lshtm.ac.uk (c) The Author(s), 2020. Published by Cambridge University Press. This is an Open Access article, distributed under the terms of the Creative Commons Attribution licence (http://creativecommons.org/licenses/by/4.0/), which permits unrestricted re-use, distribution, and reproduction in any medium, provided the original work is properly cited.

\section{CAMBRIDGE} UNIVERSITY PRESS

\title{
Bias correction methods for test-negative designs in the presence of misclassification
}

A. Endo ${ }^{1,2,3}$ (D) S. Funk ${ }^{1,2}$ and A. J. Kucharski ${ }^{1,2}$

${ }^{1}$ Department of Infectious Disease Epidemiology, London School of Hygiene \& Tropical Medicine, Keppel St. London WC1E 7HT, UK; ${ }^{2}$ Centre for the Mathematical Modelling of Infectious Diseases, London School of Hygiene \& Tropical Medicine, Keppel St., London WC1E 7HT, UK and ${ }^{3}$ The Alan Turing Institue, Euston Rd., London NW1 2DB, UK

\begin{abstract}
The test-negative design (TND) has become a standard approach for vaccine effectiveness (VE) studies. However, previous studies suggested that it may be more vulnerable than other designs to misclassification of disease outcome caused by imperfect diagnostic tests. This could be a particular limitation in VE studies where simple tests (e.g. rapid influenza diagnostic tests) are used for logistical convenience. To address this issue, we derived a mathematical representation of the TND with imperfect tests, then developed a bias correction framework for possible misclassification. TND studies usually include multiple covariates other than vaccine history to adjust for potential confounders; our methods can also address multivariate analyses and be easily coupled with existing estimation tools. We validated the performance of these methods using simulations of common scenarios for vaccine efficacy and were able to obtain unbiased estimates in a variety of parameter settings.
\end{abstract}

\section{Introduction}

Vaccine effectiveness (VE) is typically estimated as the vaccine-induced risk reduction of the target disease (TD) and has been traditionally studied using cohort or case-control designs. However, the test-negative design (TND) is becoming a popular alternative design for VE studies $[1,2]$. This is a modified version of the case-control study with an alternative definition of the control group; traditional case-control studies usually define controls as nondisease individuals in the study population, while TND studies use individuals with similar symptoms to the TD but presenting negative test results (i.e. patients of non-target diseases; ND). The TND can therefore minimise ascertainment bias by including only medicallyattended patients in both case and control groups. Many TND studies have focused on influenza vaccination, but recent studies have also considered other diseases including pneumococcal disease $[3,4]$ and rotavirus disease [5-7].

Despite its increasing popularity, a TND can be more vulnerable than other study designs to misclassification of disease outcome. Multiple studies have shown that VE is underestimated when the diagnostic tests used in the study are imperfect (i.e. have a sensitivity and/or a specificity less than 100\%) [8-10]. This can be a particular issue when simple tests (e.g. rapid diagnostic tests) are used for logistical convenience, as simple tests tend to have lower diagnostic performance than more advanced tests (e.g. polymerase chain reaction; PCR). Previous studies evaluated the expected degree of bias and concluded that specificity had a more important effect on bias than sensitivity [8-11]. These findings appear to support the use of rapid tests, despite limited sensitivity, because the specificity of these tests is typically high [2]. However, theoretical studies to date have been based on a limited range of assumptions about efficacy and pathogen epidemiology; it is therefore unclear whether such conclusions hold for all plausible combinations of scenarios.

If a study is expected to generate a non-negligible bias in estimation, such bias needs to be assessed and - if possible - corrected before the estimate is reported. Greenland [12] proposed a bias correction method for cohort studies where the sensitivity and specificity of the test are known (or at least assumed). However, it has been pointed out that bias correction in casecontrol studies is in general difficult because of differential recruitment, whereby the probability of recruiting (test-positive) cases and (negative) controls may be different [12, 13]. Although TND studies are often considered to be special cases of case-control studies, they are free from the issue of differential recruitment because the recruitment and classification are mutually-independent [14]. This means that, while Greenland's method does not apply to TND as-is, another type of bias correction may still be possible. For example, De Smedt et al. have characterised the misclassification bias in VE in the TND in a simulation study [10]. One limitation of their formulation was it relies on the unobserved âœtrueâ disease risk being known, where in reality this is not usually measurable in field studies. As a result, 
bias correction methods for TND studies that are directly applicable to field data have not yet been proposed. Moreover, previous analysis of misclassification bias has not considered the impact of multivariate analysis, where potential confounders (e.g. age and sex) are also included in the model used to estimate VE.

To address these issues, we develop a bias correction method for the test-negative VE studies that uses only data commonly available in field studies. We also apply these methods to multivariate analyses. As our approach uses the so-called multiple overimputation (MO) framework (generalisation of multiple imputation) [15], it can easily be coupled with a wide range of estimation tools without modifying their inside algorithms. Finally, we evaluate the performance of our methods by simulations of plausible epidemiological scenarios.

\section{Methods and results}

\section{Characterising bias in TND studies}

First, we consider the case where only vaccination history is included as a risk factor of acquiring the TD (i.e. the univariate setting). Following the approach of Haber et al. [16], we consider four steps in the case reporting process: vaccination, onset of symptoms, seeking of medical care and diagnosis. For simplicity, let us assume that occurrence of TD and ND are mutually independent, where their prevalences in the unvaccinated population are represented as $r_{1}$ and $r_{0}$, respectively ${ }^{1}$. Let $n_{V}$ and $n_{U}$ be the vaccinated and unvaccinated population size. The target variable in VE studies is $\gamma$, the relative risk of TD in the vaccinated population relative to the unvaccinated (i.e. $\mathrm{VE}=1-\gamma$ ). Vaccinated and unvaccinated population can have different likelihoods of seeking medical treatment given disease. We denote by $m_{V}$ and $m_{U}$ the probability of medical attendance given ND in vaccinated and unvaccinated population, respectively.

As our focus in the present study is the bias in VE estimation caused by imperfect tests, we made two key assumptions following Haber et al. [16]. One assumption is that vaccines have no effect on the risk of ND. This enables ND patients to be eligible for a control group and is a key assumption in TND studies. The other assumption is that the probability of medical attendance in vaccinated and unvaccinated population given infection is constant regardless of the disease (TD or ND). The probability of medical attendance given TD may be different from ND $\left(m_{V}\right.$ and $m_{U}$ ), potentially due to difference in severity; we assume that these probabilities are obtained by multiplying a constant factor $\mu$ (i.e. $\mu m_{V}$ and $\left.\mu m_{U}\right)^{2}$. These assumptions may not always hold and TND can be biased in such cases. However, we assume that they do in the following analysis to keep our focus on misclassification bias; namely, the study was assumed to be able to provide an unbiased VE estimate if tests are perfect.

Following the above notations, we can classify the vaccinated and unvaccinated population into multiple categories shown in Table 1. We can characterise different VE study designs (cohort, case-control and test-negative) by the categories in Table 1 from which each design tries to sample: the cohort design samples from populations $n_{V}$ and $n_{U}$ and follows them up to see what proportions fall into $x_{V}$ and $x_{V}$; the case-control design samples from

\footnotetext{
${ }^{1}$ It has been suggested that a possible violation of this assumption occur as a result of virus interference [17], but conclusive evidence for this is currently lacking $[18,19]$ and the effect on VE estimates may be limited in any case [20].

${ }^{2}$ This may not be true, for example, if vaccination reduces the severity of TD and hence reduces the likelihood of medical attendance.
}

medically-attended cases $\left(x_{V}+x_{U}\right)$ and non-diseased controls $\left(n_{V}-x_{V}+n_{U}-x_{U}\right)$ and calculate the odds ratio to approximate the relative risk (however, the actual studies can mismeasure these variables when misclassificaiton is present).

In TND studies, medically-attended patients $\left(x_{V}+x_{U}+y_{V}+\right.$ $\left.y_{U}\right)$ are sampled and classified into four categories based on the test result and vaccine history. Let $q$ be the proportion sampled relative to the population. Denoting the observed case counts with misclassification by $X$ and $Y$, the process of data collection in TND can be represented by the following matrix expression:

$$
\left[\begin{array}{ll}
X_{V} & X_{U} \\
Y_{V} & Y_{U}
\end{array}\right]=q\left[\begin{array}{ll}
\alpha & 1-\beta \\
1-\alpha & \beta
\end{array}\right]\left[\begin{array}{ll}
x_{V} & x_{U} \\
y_{V} & y_{U}
\end{array}\right]
$$

where $\alpha$ and $\beta$ are the sensitivity and specificity of the test, respectively. Matrix

$$
C=\left[\begin{array}{ll}
\alpha & 1-\beta \\
1-\alpha & \beta
\end{array}\right]
$$

describes the conversion from the true disease state to the observed result. We hereafter refer to $C$ as the classification matrix. The determinant $c=|C|=\alpha+\beta-1$ is the Youden index of the test and satisfies $0<c \leq 1$ (if $c<0$, the test is not predictive and the definitions of positive/negative should be swapped). Youden index indicates the level of information retained in the potentially misclassified test results. Youden index of 0 indicates that the information is completely lost and the test is no better than random guesses.

We define bias in the VE estimate to be the absolute difference between the (raw) estimate, derived from the misclassified observation, and the true value. Let $\delta=\left(\left(r_{1} \mu\right) / r_{0}\right)$ be the odds of the (medically-attended) TD in the unvaccinated population. Then the expected bias $B$ is given as a function of four independent parameters, $\alpha, \beta, \gamma$ and $\delta$ :

$$
\begin{aligned}
B(\alpha, \beta, \gamma, \delta) & =\mathrm{VE}_{\text {raw }}-\mathrm{VE}_{\text {true }} \\
& =\left(1-\gamma_{\text {raw }}\right)-\left(1-\gamma_{\text {true }}\right) \\
& =\gamma-\frac{[\alpha \gamma \delta+(1-\beta)][(1-\alpha) \delta+\beta]}{[(1-\alpha) \gamma \delta+\beta][\alpha \delta+(1-\beta)]} .
\end{aligned}
$$

This suggests that the influence of sensitivity/specificity on the degree of bias varies depending on the case ratio $\delta /(1+\delta)$, i.e. the ratio between the incidence of medical attendance for TD and ND in the unvaccinated study population ${ }^{3}$ (Fig. 1). The degree of bias also depends on $\gamma$ but is independent of $m_{V}$ and $m_{U}$. The degree of bias is largely determined by the test specificity when the case ratio is small, but the influence of sensitivity and specificity is almost equivalent to a case ratio of 0.6 . It is notable that high specificity does not always assure that the bias is negligible. This may be true if specificity is strictly $100 \%$ and the case ratio is low to moderate, but a slight decline to $97 \%$ can cause a bias up to $10-15$ percentage points. The effect of sensitivity is also non-negligible when the case ratio is high.

When the expected bias is plotted against the case ratio with various combinations of test performance, we find that VE estimates can be substantially biased for certain case ratios (especially

${ }^{3}$ For example, a case ratio of 0.5 indicates TD:ND $=1: 1$ in the unvaccinated. The value is smaller than 0.5 when $\mathrm{TD}<\mathrm{ND}$ and greater than 0.5 when $\mathrm{TD}>\mathrm{ND}$. 
Table 1. Population classified into different categories of interest in VE studies

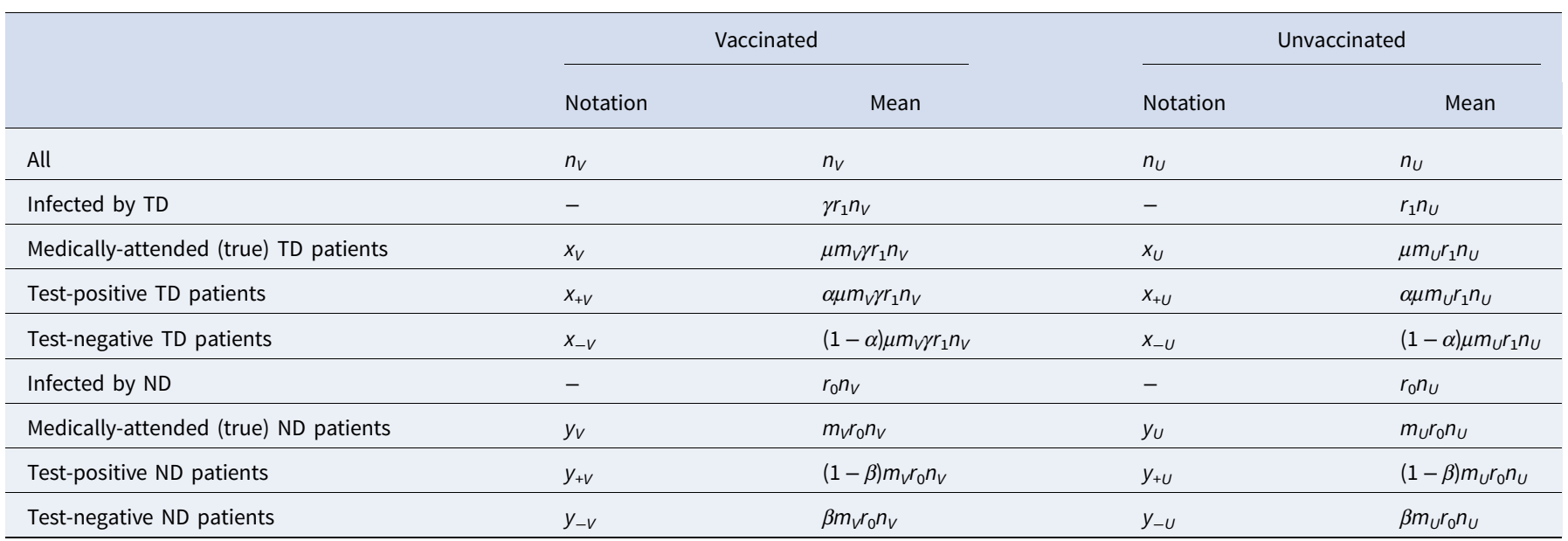

(a)

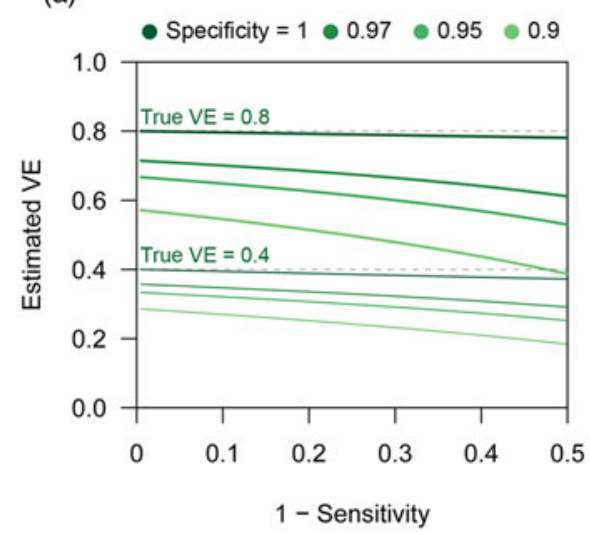

(d)

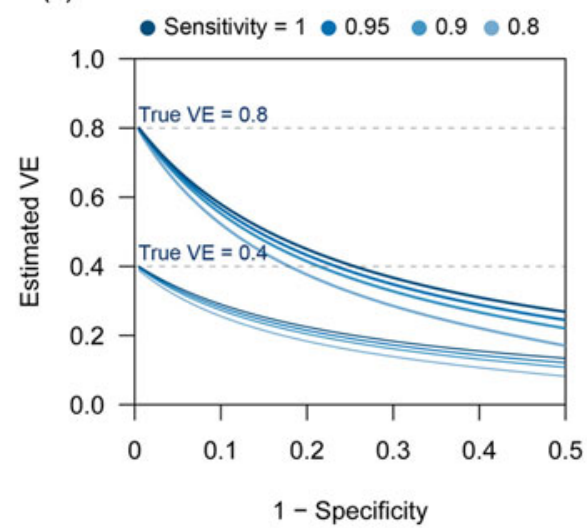

(b)

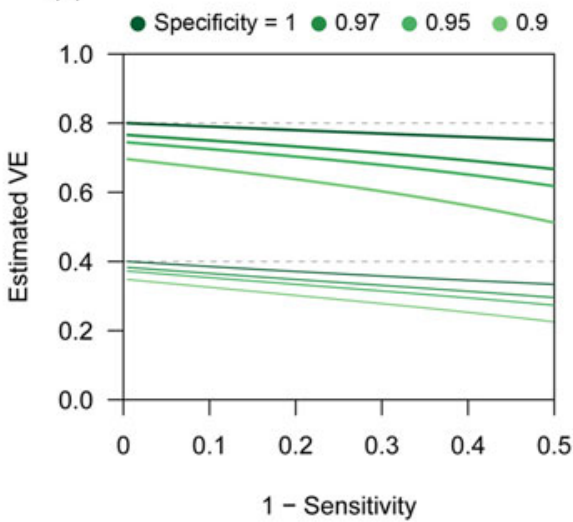

(e)

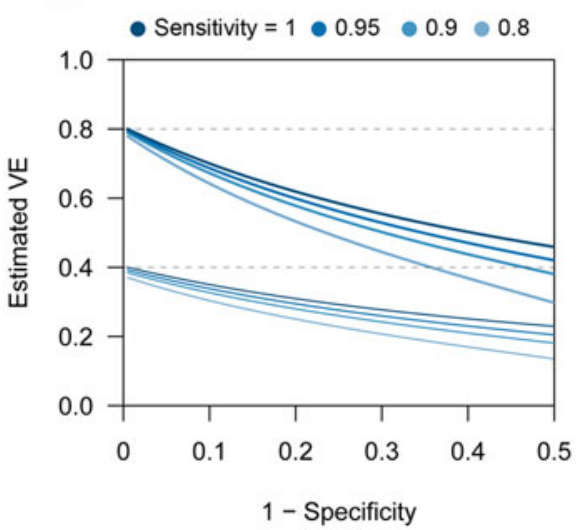

(c)

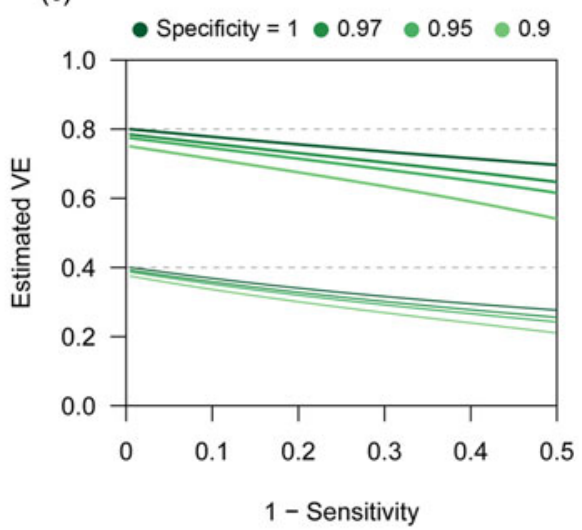

$(f)$

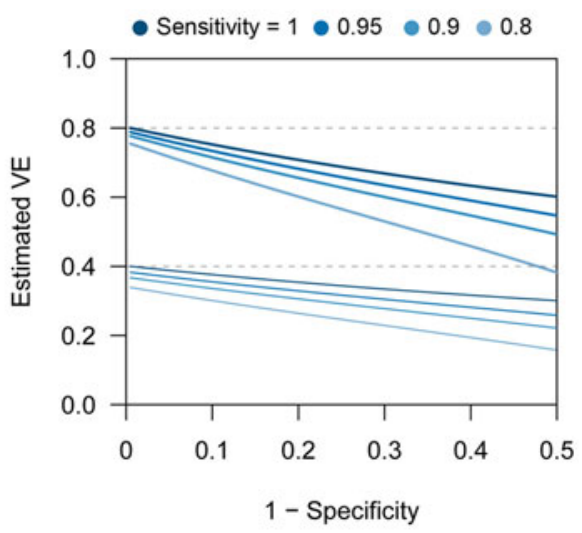

Fig. 1. Bias in VE estimates caused by misclassification for different combinations of parameter values. (a)-(c) Estimated VE plotted against sensitivity. (a) True case ratio (the true ratio between TD and ND cases included in the study) $=0.2$ (b) 0.4 (c) 0.6 . Each two sets of lines respectively correspond to different true VEs ( $80 \%$ and $40 \%$, denoted by the dotted lines). (d)-(f) Estimated VE plotted against specificity. (d) True case ratio $=0.2$ (e) 0.4 (f) 0.6 .

when the ratio is far from 1:1), even with reasonably high sensitivity and specificity (Fig. 2b). In TND studies, researchers have no control over the case ratio because the study design requires that all tested individuals be included in the study. We found that the proportion of TD-positive patients in previous TND studies (retrieved from three systematic reviews [21-23]) varied considerably, ranging from $10 \%$ to $70 \%$ (Fig. $2 \mathrm{a}$ ) ${ }^{4}$. Because of this large variation in the case ratio, it would be difficult to predict the degree of bias before data collection. Post-hoc assessment and

${ }^{4}$ Strictly speaking, proportion positive is a different quantity from case ratio, but it should serve as a reasonable proxy of the case ratio in most settings. 
(a)

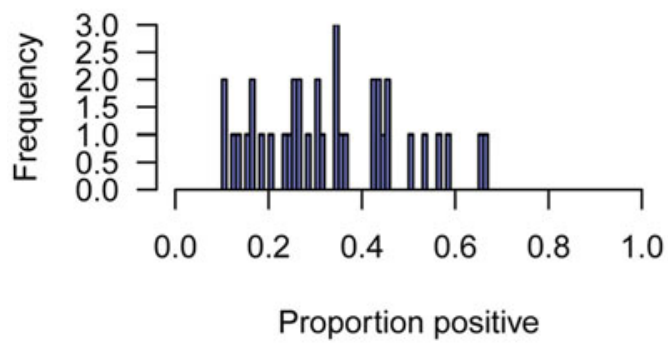

(b)

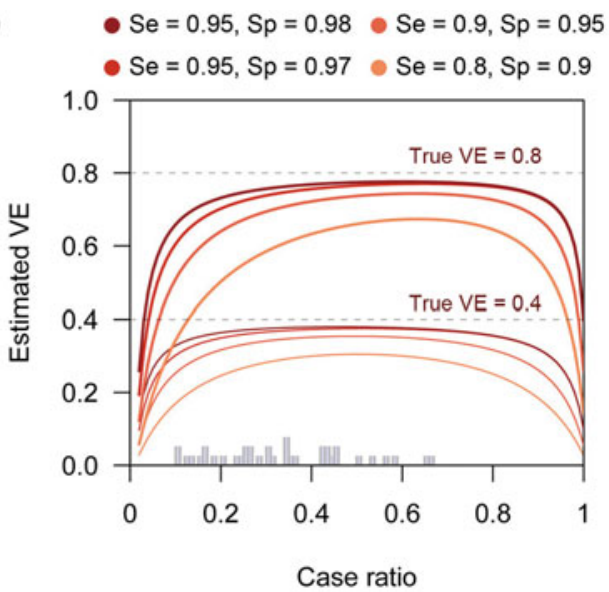

Fig. 2. Biased VE estimates with varying case ratio and the observed proportion of positive patients. (a) The proportion of test-positive patients in TND studies from systematic reviews. The proportions were retrieved from three systematic reviews [21-23]. (b) Estimated VE plotted against case ratio. Two sets of lines respectively correspond to different true VEs ( $80 \%$ and $40 \%$, denoted by the dotted lines). The histogram in Panel (a) is overlaid on the $x$-axis.

correction therefore need to be considered. See the Supplementary Document for further analysis of the degree of bias.

\section{Bias correction in univariate analysis}

\section{Corrected odds ratio}

Although TND has sometimes been referred to as a special case of the case-control design, there is a distinct feature in the sampling procedure of TND. It has been pointed out that the adjustment methods for misclassification bias developed for cohort studies do not apply to case-control studies because the sampling ratio in case-control studies (differential recruitment) varies between case and control groups $[12,13]$. However, as we have shown in Section 'Characterising bias in TND studies', both cases (TD patients) and controls (ND patients) are sampled at the same ratio $(q)$ in TND studies. This suggests that the existing bias correction formulas developed for a hypothetical setting [13] where the whole population is evenly sampled (which is unrealistic in traditional studies) may be applicable to TND studies.

By left-multiplying Equation (1) with the inverted matrix $C^{-1}$, we can obtain the corrected odds ratio $\gamma^{\star}$ as

$$
\gamma^{*}=\frac{X_{V}-((1-\beta) / \beta) Y_{V}}{Y_{V}-((1-\alpha) / \alpha) X_{V}} \cdot \frac{Y_{U}-((1-\alpha) / \alpha) X_{U}}{X_{U}-((1-\beta) / \beta) Y_{U}}
$$

which adjusts for misclassification to give an asymptoticallyunbiased estimate of $\gamma$. This result can also be derived by maximising the likelihood accounting for misclassification in the observed TND data (see the Supplementary Document).

All four components of (3) (two numerators and two denominators) are usually expected to be non-negative with moderate VE (less than 100\%) because these components are considered to be proportional to reconstructed true case counts. However, in some (relatively rare) cases, one or more components may become negative due to random fluctuations in observation. Theoretically, negative values are not permitted as true case counts, and thus such negative quantities would need to be truncated to 0 . As a result, the corrected odds ratio can be either 0 or infinity. It is unrealistic in clinical settings that vaccines have absolute $100 \%$ or $-100 \%$ effectiveness. Uncertainty around such MLEs should be carefully considered; increasing sample size or redesigning the study might be recommended where possible. Alternatively, the Bayesian framework may be used to yield an interval estimate with the likelihood shown in section 'Direct likelihood method for the logistic regression model' adapted for a univariate model.

The confidence interval for VE can be obtained by assuming $\log$-normality of the odds ratio $\gamma$, i.e.

$$
\gamma=\gamma^{*} \exp \left( \pm 1.96 \sigma^{*}\right)
$$

where $\sigma$ is the shape parameter of the log-normal distribution and is empirically given as

$$
\begin{aligned}
& \sigma^{*}=S D\left(\log \left(\gamma^{*}\right)\right) \\
&=c \sqrt{\frac{X_{V} Y_{V}\left(X_{V}+Y_{V}\right)}{\frac{\left.\alpha Y_{V}-(1-\alpha) X_{V}\right]^{2}\left[\beta X_{V}-(1-\beta) Y_{V}\right]^{2}}{X_{U} Y_{U}\left(X_{U}+Y_{U}\right)}}} . \\
&
\end{aligned}
$$

See the Supplementary Document for details of the MLE and confidence intervals.

\section{Simulation}

To assess the performance of the corrected odds ratio given in Equation (3) and uncertainty around it, we used simulation studies. TND study datasets were drawn from Poisson distributions (see the Supplementary Document for model settings and the likelihood function) as it is a reasonable assumption when medicallyattended cases are recruited over the study period. We parameterised the mean incidence in the dataset by the 'baseline medical attendance' $\lambda_{V}=q m_{V}\left(r_{1} \mu+r_{0}\right) n_{V}$ and $\lambda_{U}=q m_{U}\left(r_{1} \mu+r_{0}\right) n_{U}$, so that $\lambda_{V}$ and $\lambda_{U}$ correspond to the mean number of vaccinated/ unvaccinated patients when vaccine has no effect (i.e. $\gamma=1$ and $\mathrm{VE}=0)$. The mean total sample size (given as $((1+\gamma \delta) /(1+\delta))$ $\left.\lambda_{V}+\lambda_{U}\right)$ was set to be 3000 . Parameter values were chosen according to a range of scenarios shown in Table 2, and the true $\mathrm{VE}=1$ $-\gamma$ was compared with the estimates obtained from the simulated data. For each scenario, simulation was repeated 500 times to yield the distribution of estimates. Reproducible codes (including those 
Table 2. Simulation settings

\begin{tabular}{|c|c|c|c|c|c|c|}
\hline ID & Scenario & True VE $(\gamma)$ & $\lambda_{V} / \lambda_{U}$ & Case ratio $(\gamma /(1+\gamma))$ & Sensitivity $(\alpha)$ & Specificity $(\beta)$ \\
\hline 1 & Baseline: low VE & 0.4 & 0.5 & 0.5 & 0.8 & 0.95 \\
\hline 2 & Baseline: high VE & 0.8 & 0.5 & 0.5 & 0.8 & 0.95 \\
\hline 3 & High quality test: low VE & 0.4 & 0.5 & 0.5 & 0.95 & 0.97 \\
\hline 4 & High quality test: high VE & 0.8 & 0.5 & 0.5 & 0.95 & 0.97 \\
\hline 5 & Low quality test: low VE & 0.4 & 0.5 & 0.5 & 0.6 & 0.9 \\
\hline 6 & Low quality test: high VE & 0.8 & 0.5 & 0.5 & 0.6 & 0.9 \\
\hline 7 & High TD incidence: low VE & 0.4 & 0.5 & 0.7 & 0.8 & 0.95 \\
\hline 8 & High TD incidence: high VE & 0.8 & 0.5 & 0.7 & 0.8 & 0.95 \\
\hline 9 & Low TD incidence: low VE & 0.4 & 0.5 & 0.3 & 0.8 & 0.95 \\
\hline 10 & Low TD incidence: high VE & 0.8 & 0.5 & 0.3 & 0.8 & 0.95 \\
\hline 11 & High vaccine coverage: low VE & 0.4 & 0.7 & 0.5 & 0.8 & 0.95 \\
\hline 12 & High vaccine coverage: high VE & 0.8 & 0.7 & 0.5 & 0.8 & 0.95 \\
\hline 13 & Low vaccine coverage: low VE & 0.4 & 0.3 & 0.5 & 0.8 & 0.95 \\
\hline 14 & Low vaccine coverage: high VE & 0.8 & 0.3 & 0.5 & 0.8 & 0.95 \\
\hline
\end{tabular}
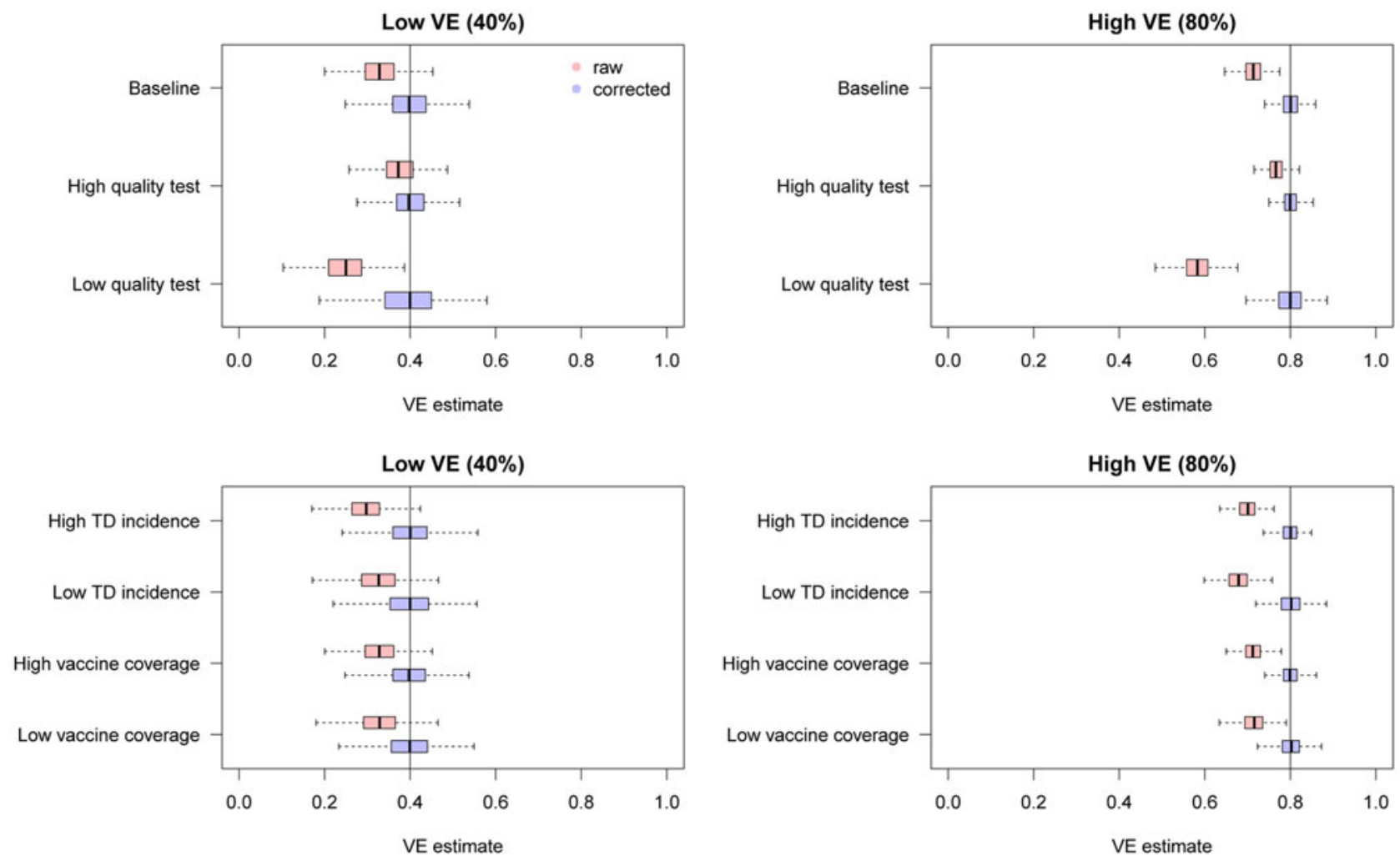

Fig. 3. Bias correction for simulated data in the univariate setting. The distributions of bias-corrected VE estimates (boxplots in blue) are compared with those of raw VE estimates without correction (red). Five hundred independent datasets were randomly generated for each set of parameter values, and the corrected and uncorrected VE estimates are compared with the true value (black solid line). See Table 2 for parameter settings in each scenario.

for simulations in later sections) are reposited on GitHub (https:// github.com/akira-endo/TND-biascorrection/).

We found that the uncorrected estimates, directly obtained from the raw case counts that were potentially misclassified, exhibited substantial underestimation of VE for most parameter values (Fig. 3). On the other hand, our bias correction method was able to yield unbiased estimates in every setting, whose median almost correspond to the true VE. Although the corrected and uncorrected distributions were similar (with a difference in median $\sim 5 \%$ ) when VE is relatively low (40\%) and the test has sufficiently high sensitivity and specificity $(95 \%$ and $97 \%$, respectively), they became distinguishable with a higher VE (80\%). With 
lower test performances, the bias in the VE estimates can be up to $10-20 \%$, which may be beyond the level of acceptance in VE studies.

\section{Bias correction of VEs reported in previous studies}

We have seen that the degree of bias for uncorrected VE estimates depends on parameter values. To explore the possible degree of bias in existing VE studies, we extracted the reported crude VEs (i.e. VEs unadjusted for potential confounders) from two systematic reviews $[21,23]^{5}$ and applied our bias correction method assuming different levels of test sensitivity and specificity. The case counts for each study summarised in the reviews were considered eligible for the analysis if the total sample size exceeded 200. Varying the assumed sensitivity and specificity, we investigated the possible discrepancy between the reported VE (or crude VE derived from the case counts if unreported in the reviews) and bias-corrected VE. We did not consider correcting adjusted VEs because it requires access to the original datasets.

Figure 4 displays the discrepancy between the reported VE and bias-corrected VE corresponding to a range of assumptions on the test performance. Many of the extracted studies employed PCR for the diagnostic test, which is expected to have a high performance. However, the true performance of PCR cannot be definitively measured as there is currently no other gold-standard test available. Figure 4 suggests that even a slight decline in the test performance can introduce a non-negligible bias in some parameter settings. Our bias correction methods may therefore also be useful in TND studies using PCR, which would enable a sensitivity analysis accounting for potential misdiagnosis by PCR tests. In this light, it is useful that the corrected odds ratio

$$
\gamma^{*}=\frac{X_{V}-((1-\beta) / \beta) Y_{V}}{Y_{V}-((1-\alpha) / \alpha) X_{V}} \cdot \frac{Y_{U}-((1-\alpha) / \alpha) X_{U}}{X_{U}-((1-\beta) / \beta) Y_{U}}
$$

is a monotonic function of both $\alpha$ and $\beta$ (given that all the four components are positive). The possible range of VE in a sensitivity analysis is obtained by supplying $\gamma^{\star}$ with the assumed upper/ lower limits of sensitivity and specificity.

\section{Bias correction in multivariate analysis}

\section{Theoretical framework}

TND studies often employ a multivariate regression framework to address potential confounding variables such as age. The most widespread approach is to use generalised linear models (e.g. logistic regression) and include vaccination history as well as other confounding variables as covariates. The estimated linear coefficient for vaccination history can then be converted VE (in the logistic regression model, the linear coefficient for vaccination history corresponds to $\log (1-\mathrm{VE}))$. In this situation, the likelihood function now reflects a regression model and thus the biascorrected estimate in the univariate analysis (Equation (3)) is no longer applicable. We therefore need to develop a separate multivariate TND study framework to correct for bias in multivariate analysis.

Suppose that covariates $\xi=\left(\xi^{1}, \xi^{2}, \ldots, \xi^{n}\right)$ are included in the model, and that $\xi^{1}$ corresponds to vaccination history (1: vaccinated, 0: unvaccinated). These covariates $\xi_{i}$, as well as outcome variable $Z_{i}$ (i.e. test results) are available for each individual $i$ included in the study. In TND studies, it is often convenient to

${ }^{5}$ Young et al. [22] was not included because they did not report case counts.

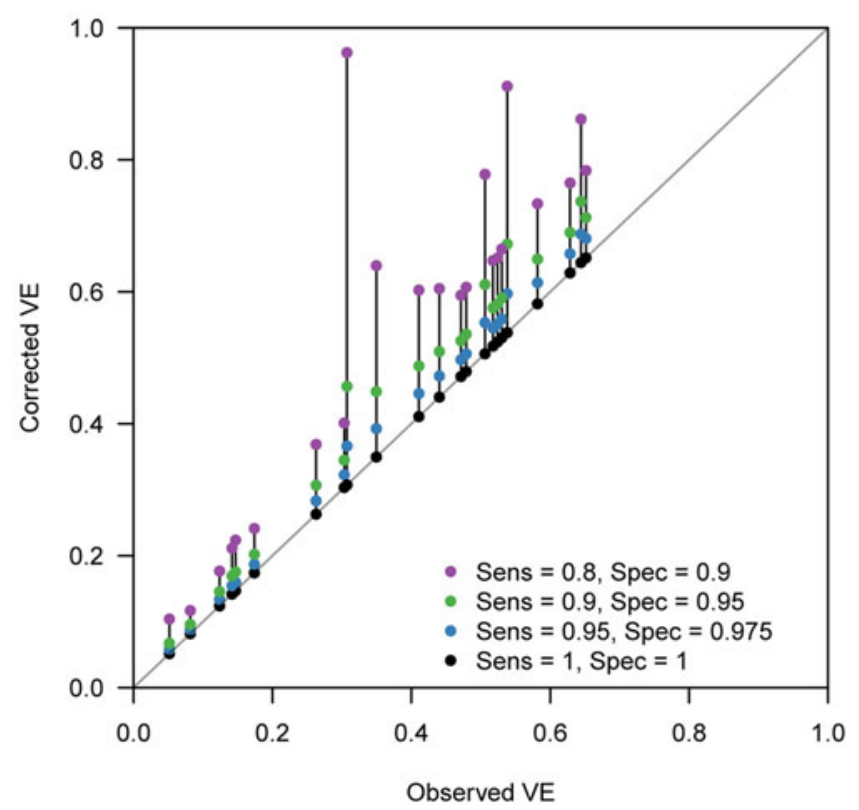

Fig. 4. Bias correction method applied to published VE estimates assuming various test sensitivity and specificity. Case count data were extracted from two systematic reviews [21, 23]. Each connected set of dots show how (crude) VE estimates reported in the review varies when imperfect sensitivity and specificity are assumed. Black dots on the grey diagonal line denote the original VEs reported in the reviews. This should correspond to the true value if sensitivity $=$ specificity $=1$. Coloured dots show the bias-corrected VE considering potential misclassification.

model the binomial probability for the true outcome $p_{1}\left(\xi_{i}\right)$, i.e. the conditional probability that the true outcome is TD as opposed to ND given an individual has covariates $\xi_{i}$. Let us use parameter set $\theta$ to model the binomial probabilities $p_{1}$ (and $\left.p_{0}=1-p_{1}\right)$. Using the binomial probability $\pi_{Z_{i}}$ for observed (potentially misclassified) outcome $Z_{i}$, we can obtain the MLE for $\theta$ by maximising

$$
\begin{aligned}
\mathcal{L}(\theta ; D)= & \prod_{S}^{i=1} \pi_{Z_{i}}\left(\xi_{i} ; \theta\right) \\
& =\prod_{i \in\{+\}}\left[\alpha p_{1}\left(\xi_{i} ; \theta\right)+(1-\beta) p_{0}\left(\xi_{i} ; \theta\right)\right] \\
& \times \prod_{i \in\{-\}}\left[(1-\alpha) p_{1}\left(\xi_{i} ; \theta\right)+\beta p_{0}\left(\xi_{i} ; \theta\right)\right] .
\end{aligned}
$$

With the estimate $\theta^{*}$, the VE estimate for an individual with covariates $\xi^{2: n}=\left(\xi^{2}, \xi^{3}, \ldots, \xi^{n}\right)$ is given as (1-odds ratio):

$$
\operatorname{VE}\left(\xi^{2: n}\right)=1-\frac{p_{1}\left(\xi^{1}=1, \xi^{2: n} ; \theta^{*}\right)}{p_{0}\left(\xi^{1}=1, \xi^{2: n} ; \theta^{*}\right)} / \frac{p_{1}\left(\xi^{1}=0, \xi^{2: n} ; \theta^{*}\right)}{p_{0}\left(\xi^{1}=0, \xi^{2: n} ; \theta^{*}\right)}
$$

See the Supplementary Document for further details.

\section{Direct likelihood method for the logistic regression model}

The logistic regression model is well-suited for modelling binomial probabilities $p_{1}$ and $p_{0}$. The $\log$-odds $\left(\log \left(\frac{p_{1}}{p_{0}}\right)\right)$ is characterised by a linear predictor as:

$$
\log \left(\frac{p_{1}(\xi ; \theta)}{p_{0}(\xi ; \theta)}\right)=\theta_{0}+\theta_{1} \xi^{1}+\cdots+\theta_{n} \xi^{n}
$$


In the logistic regression model where covariate $\xi_{1}$ indicates vaccination history, the corresponding coefficient $\theta_{1}$ gives the VE estimate: $\mathrm{VE}=1-\exp \left(\theta_{1}\right)$. Due to the assumed linearity, the estimated VE value is common across individuals regardless of covariates $\xi^{2: n}$.

We can employ the direct likelihood method by combining Equations (5) and (7). The usual logistic regression optimises $\theta$ by assuming that the test results follow Bernoulli distributions $Z_{i} \sim \operatorname{Bernoulli}\left(p_{1}\left(\xi_{i} ; \theta\right)\right)\left(Z_{i}=1\right.$ for positive test results and 0 for negative). To correct the misclassification bias, we instead need to use the modified probabilities to construct the likelihood accounting for diagnostic error, i.e.

$$
\begin{aligned}
Z_{i} & \sim \operatorname{Bernoulli}\left(\pi_{+}\left(\xi_{i} ; \theta\right)\right) \\
& =\operatorname{Bernoulli}\left(\alpha p_{1}\left(\xi_{i} ; \theta\right)+(1-\beta) p_{0}\left(\xi_{i} ; \theta\right)\right) .
\end{aligned}
$$

Parameter $\theta$ is estimated by directly maximising the probability of observing $\left\{Z_{i}\right\}$ based on Equation (8)

Note that as long as the binomial probability is the modelling target, other type of models (e.g. machine learning classifiers) could also be employed under a similar framework.

\section{Multiple overimputation combined with existing tools}

The direct likelihood method presented in the previous section is the most rigorous MLE approach and would therefore be preferable whenever possible. However, it is often technicallydemanding to implement such approaches as it involves re-defining the likelihood; if we wanted to use existing tools for logistic regression (or other models), for example, we would need to modify the internal algorithm of such tools. This is in particular complicated in tools for generalised linear models including logistic regression, whose standard algorithm is the iteratively reweighted least squares method [24], which does not involve the explicit likelihood. To ensure that our correction methods can be employed without losing access to substantial existing software resources, we also propose another method, which employs a MO framework [15] to account for misclassification. Whereas multiple imputation only considers missing values, $\mathrm{MO}$ is proposed as a more general concept which includes overwriting mismeasured values in the dataset by imputation. In our multivariate bias correction method, test results in the dataset (which are potentially misclassified) are randomly overimputed.

Let $M$ be an existing estimation software tool whose likelihood specification cannot be reprogrammed. Given data $d=\left\{z_{i}, \xi_{i}\right\}_{i=1,2, \ldots S}$, where $z_{i}$ denotes the true disease state $(z=1$ for TD and $z=0$ for $\mathrm{ND}), M$ would be expected to return at least the following two elements: the point estimate of $\mathrm{VE}\left(\varepsilon_{d}\right)$ and the predicted binomial probability $\hat{p}_{1}\left(\xi_{i}\right)$ for each individual $i$. From the original observed dataset $D, J$ copies of imputed datasets $\left\{\tilde{D}^{j}\right\}=\left\{\tilde{D}^{1}, \tilde{D}^{2}, \ldots, \tilde{D}^{J}\right\}$ are generated by the following procedure.

(1) For $i=1,2, \ldots, S$, impute disease state $\tilde{z}_{i}^{j}$ based on the test result $Z_{i}$. Each $\tilde{z}_{i}^{j}$ is sampled from a Bernoulli distribution conditional to $Z_{i}$ :

$$
\tilde{z}_{i}^{j} \sim\left\{\begin{array}{ll}
\operatorname{Bernoulli}\left(1-\tilde{\varphi}_{i+}\right) & \left(Z_{i}=1\right) \\
\operatorname{Bernoulli}\left(\tilde{\varphi}_{i-}\right) & \left(Z_{i}=0\right)
\end{array} .\right.
$$

(2) $\tilde{\varphi}_{i+}$ and $\tilde{\varphi}_{i-}$ are estimated probabilities that the test result for individual $i$ is incorrect (i.e. $z_{i} \neq Z_{i}$ ) given $Z_{i}$. The sampling procedure (9) is therefore interpreted as the test result $Z_{i}$ being 'flipped' at a probability $\tilde{\varphi}_{i+}$ or $\tilde{\varphi}_{i-}$. Later we will discuss possible procedures to obtain these probabilities.

(3) Apply $M$ to $\tilde{D}^{j}=\left\{\tilde{z}_{i}^{j}, \xi_{i}\right\}$ to yield a point estimate of $\operatorname{VE}\left(\varepsilon^{j}\right)$.

(4) Repeat (1) and (2) for $j=1,2, \ldots, J$ to yield MO estimates $\left\{\varepsilon^{j}\right\}_{j=1, \ldots J}$.

Once MO estimates $\left\{\varepsilon^{j}\right\}$ are obtained, the pooled estimate and confidence intervals of VE are obtained by appropriate summary statistics, e.g. Rubin's rules [25]. As long as the estimated 'flipping' probabilities $\tilde{\varphi}_{i \pm}=\left(\tilde{\varphi}_{i+}, \tilde{\varphi}_{i-}\right)$ are well chosen, this MO procedure should provide an unbiased estimate of VE with a sufficiently large number of iterations $J$.

As a method to estimate the flipping probability $\tilde{\varphi}_{i \pm}$, here we propose the parametric bootstrapping described as follows. Given the observed test result $Z_{i}, \tilde{\varphi}_{i \pm}$ is given as the Bayesian probability

$\mathcal{P}\left(z_{i}=Z_{i} \mid Z_{i}\right)$

$=\left\{\begin{array}{c}\frac{\alpha \mathcal{P}\left(z_{i}=1\right)}{\alpha \mathcal{P}\left(z_{i}=1\right)+(1-\beta) \mathcal{P}\left(z_{i}=0\right)}=\frac{\alpha p_{1}\left(\xi_{i}\right)}{\alpha p_{1}\left(\xi_{i}\right)+(1-\beta) p_{0}\left(\xi_{i}\right)}\left(Z_{i}=1\right) \\ \frac{\beta \mathcal{P}\left(z_{i}=0\right)}{(1-a) \mathcal{P}\left(z_{i}=1\right)+\beta \mathcal{P}\left(z_{i}=0\right)}=\frac{\beta p_{0}\left(\xi_{i}\right)}{(1-\alpha) p_{1}\left(\xi_{i}\right)+\beta p_{0}\left(\xi_{i}\right)}\left(Z_{i}=0\right)\end{array}\right.$

Although the true binomial probabilities $p_{0}\left(\xi_{i}\right), p_{1}\left(\xi_{i}\right)$ are unknown, their estimators are derived with the inverted classification matrix in the same manner as Equation (3). By substituting

$$
\left[\begin{array}{l}
p_{1}\left(\xi_{i}\right) \\
p_{0}\left(\xi_{i}\right)
\end{array}\right]
$$

with

$$
C^{-1}\left[\begin{array}{c}
\pi_{+}\left(\xi_{i}\right) \\
\pi_{-}\left(\xi_{i}\right)
\end{array}\right]
$$

we get

$$
\begin{aligned}
& \tilde{\varphi}_{i+}=1-\mathcal{P}\left(z_{i}=1 \mid Z_{i}=1\right)=\frac{1-\beta}{\alpha+\beta-1}\left[\alpha \cdot \frac{\pi_{-}\left(\xi_{i}\right)}{\pi_{+}\left(\xi_{i}\right)}-(1-\alpha)\right] \\
& \tilde{\varphi}_{i-}=1-\mathcal{P}\left(z_{i}=0 \mid Z_{i}=0\right)=\frac{1-\alpha}{\alpha+\beta-1}\left[\beta \cdot \frac{\pi_{+}\left(\xi_{i}\right)}{\pi_{-}\left(\xi_{i}\right)}-(1-\beta)\right]
\end{aligned}
$$

These probabilities can be computed provided the odds of the test results $\pi_{+}\left(\xi_{i}\right) / \pi_{-}\left(\xi_{i}\right)$. We approximate this odds by applying estimation tool $M$ to the original data $D$; i.e. the predicted binomial probability $\hat{p}_{1}\left(\xi_{i}\right)$ obtained from $D$ is used as a proxy of $\pi_{+}\left(\xi_{i}\right)$. Generally it is not assured that true and observed probabilities $p_{1}\left(\xi_{i}\right)$ and $\pi_{+}\left(\xi_{i}\right)$ have the same mechanistic structure captured by $M$; however, when our concern is limited to the use of modelpredicted probabilities to smooth the data $D$, we may expect for $M$ to provide a sufficiently good approximation. The above framework can be regarded as a variant of parametric bootstrapping methods as $\mathrm{MO}$ datasets are generated from data $D$ assuming a parametric model $M$. The whole bias correction procedure is presented in pseudocode (Fig. 5); sample R code is also available on GitHub (https://github.com/akira-endo/TND-biascorrection/).

\section{EM algorithm}

Another possible approach to addressing misclassification is the use of the EM algorithm, which has been proposed for case- 


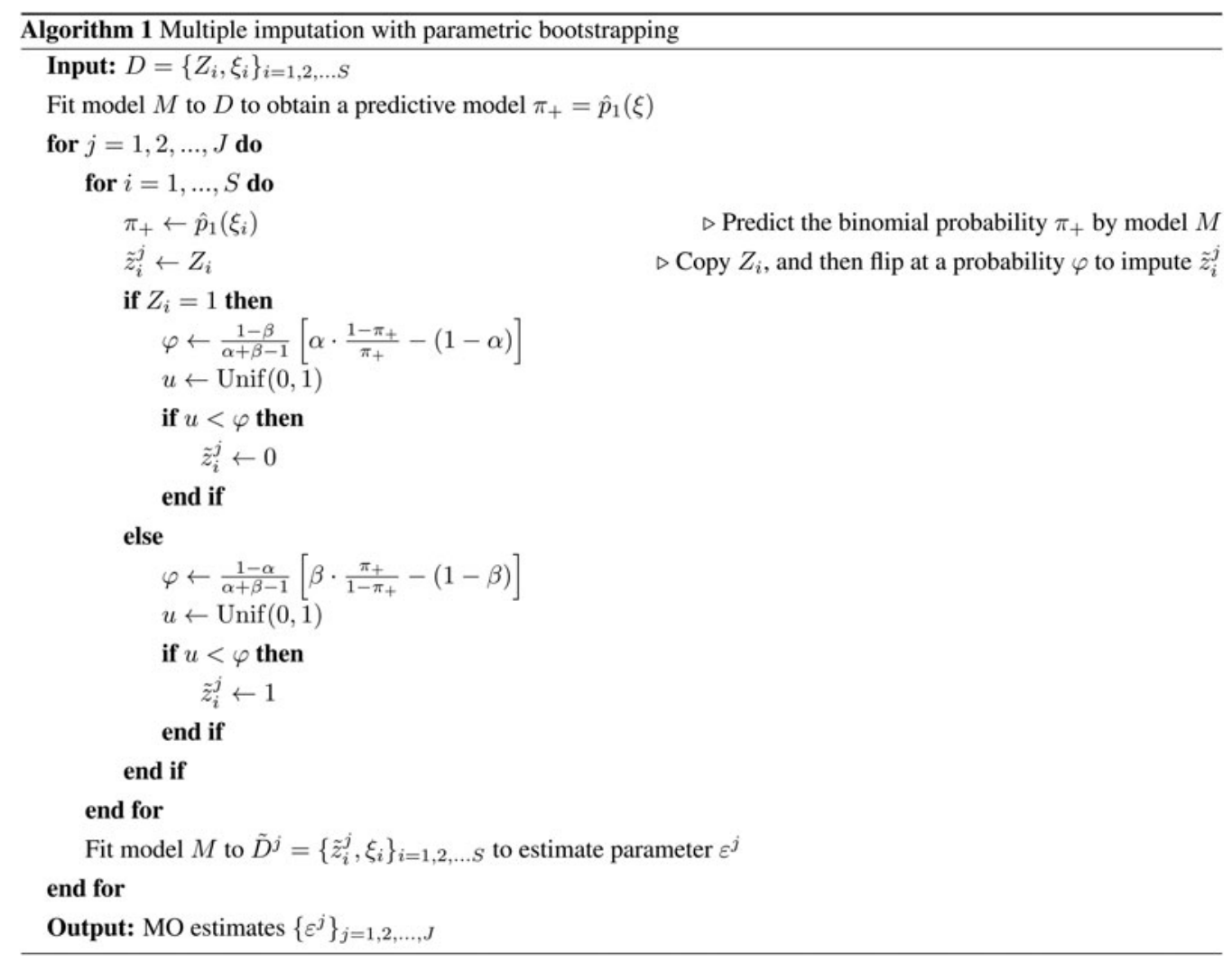

Fig. 5. Multiple imputation with parametric bootstrapping.

control studies in a previous study (where differential recruitment was not considered) [26]. Because of its methodological similarity, the algorithm can also be applied to the TND. The original EM algorithm presented in [26] would produce, if properly implemented, the result equivalent to the direct likelihood approach. However, the original EM algorithm requires that the model can handle non-integer sample weights (which may not always be assured). Moreover, computing confidence intervals in EM algorithm can be complicated. We therefore recommend parametric bootstrapping as the first choice of bias correction method when the direct likelihood approach is inconvenient.

\section{Simulation of bias correction with parametric bootstrapping}

To assess the performance of this method, we used the same simulation framework as in the univariate analysis (Table 2). In addition to vaccination history (denoted by $\xi^{1}$ ), we consider one categorical and one continuous covariate. Let us assume that $\xi_{2}$ represents the age group (categorical; 1: child, 0 : adult) and $\xi_{3}$ the pre-infection antibody titre against TD (continuous). Suppose that the population ratio between children and adults is $1: 2$, and that $\xi_{3}$ is scaled so that it is standard normally distributed in the population. For simplicity, we assumed that all the covariates are mutually independent with regard to the distribution and effects (i.e. no association between covariates and no interaction effects). The relative risk of children was set to be 2 and 1.5 for TD and ND, respectively, and a unit increase in the antibody titre was assumed to halve the risk of TD (and not to affect the risk of ND). The mean total sample size $\lambda$ was set to be 3000 , and 500 sets of simulation data were generated for each scenario. VE estimates were corrected by the parametric bootstrapping approach (the number of iterations $J=100$ ) and were compared with the raw (uncorrected) VE estimates.

Figure 6 shows the distributions of estimates with and without bias correction in the multivariate setting. Our bias correction (parametric bootstrapping) provided unbiased estimates for all the scenarios considered. Overall, biases in the uncorrected estimates were larger than those in the univariate setting. In some scenarios, the standard error of the bias-corrected estimates was extremely wide. This was primarily because of the uncertainty already introduced before misclassification rather than the failure of bias correction (as can be seen in the Supplementary Fig. S3). Larger sample size is required to yield accurate estimates in those settings, as the information loss due to misclassification will be added on top of the inherent uncertainty in the true data.

\section{The number of confounding variables}

We investigated how the bias in uncorrected VE estimates can be affected by the number of confounding variables. In addition to the vaccine history $\xi^{1}$, we added a set of categorical/continuous confounding variables to the model and assessed the degree of bias caused by misclassification. The characteristics of the variables were inherited from those in section 'Simulation of bias correction with parametric bootstrapping': categorical variable 'age' and continuous variable 'pre-infection antibody titre'. That is, individuals were assigned multiple covariates (e.g. 'categorical variable A', 'categorical variable B', ..., 'continuous variable A', 'continuous variable B', ...) whose distribution and effect were identical to 'age' (for categorical variables) and 'antibody titre' (for continuous variables) in section 'Simulation of bias correction with parametric bootstrapping'. No interaction between 

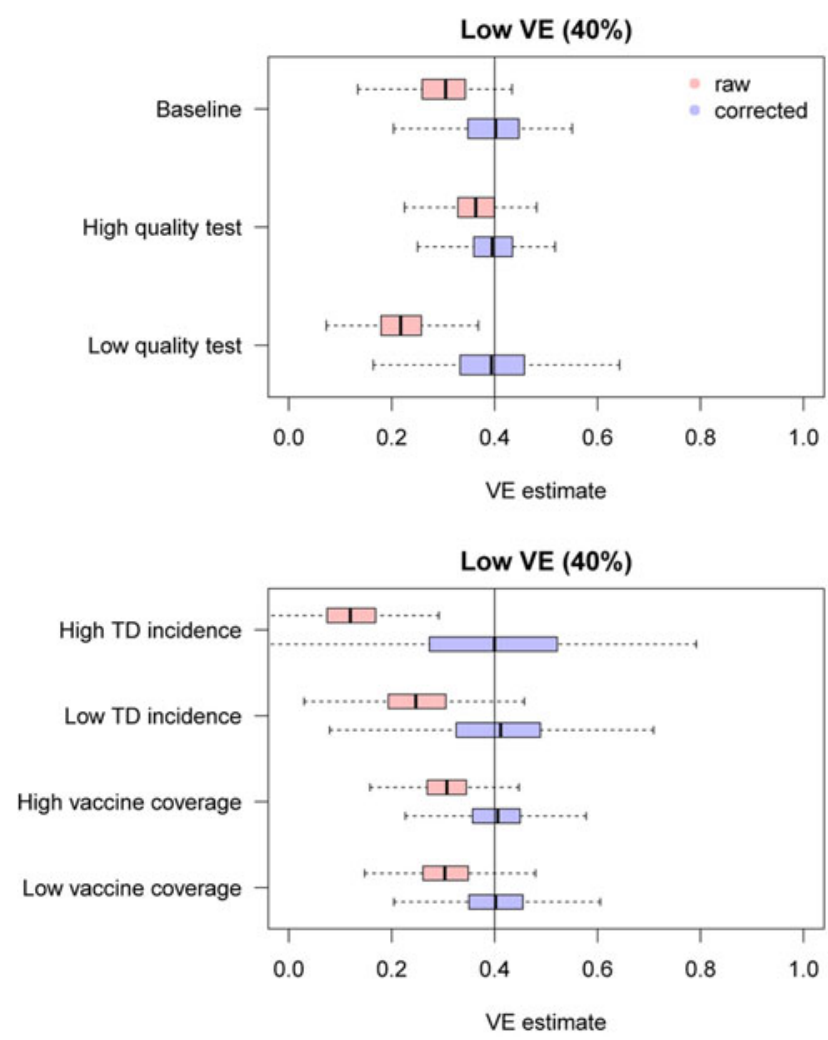
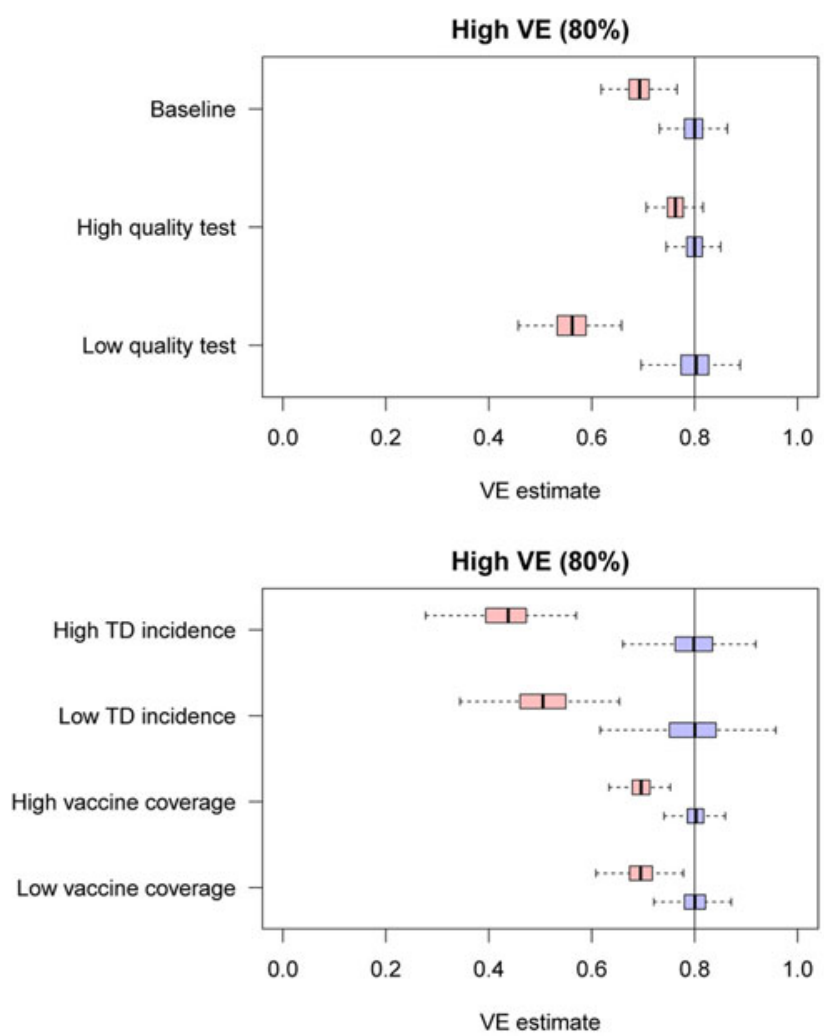

Fig. 6. Bias correction for simulated data in the multivariate setting. The distributions of bias-corrected (blue) and uncorrected (red) VE estimates from 500 simulations are compared. Dotted lines denote median and black solid lines denote the true VE. The parametric bootstrapping bias correction method was used for bias correction.

covariates was assumed. The covariate set in section 'Simulation of bias correction with parametric bootstrapping' being baseline (the number of covariates: (vaccine, categorical, continuous) $=$ $(1,1,1))$, we employed two more scenarios with a larger number of covariates: $(1,3,3)$ and $(1,5,5)$.

The simulation results are presented in Figure 7. Overall, additional confounding variables led to more severe bias in the uncorrected VE estimates towards underestimation. These results further highlight the importance of bias correction when heterogeneous disease risks are expected; VE estimates adjusted for many confounding variables can exhibit substantial misclassification bias.

\section{Discussion}

Misclassification caused by imperfect diagnostic tests can potentially lead to substantial biases in TND studies. By considering the processes involved in VE estimation, we have characterised the degree of bias potentially caused by misclassification in different parameter settings, finding that VE can be noticeably underestimated, particularly when the ratio between TD and ND cases in the study data is unbalanced. To address this potential bias, we developed multiple bias correction methods that provide unbiased VE estimates in both univariate and multivariate settings. When the test sensitivity and specificity are known or assumed, those values can be used to restore the true VE estimate by a relatively simple statistical procedure. Using simulations, we showed that our methods could successfully eliminate the bias in VE estimates obtained from misclassified data, although some uncertainty was introduced as a result of the information loss.
We believe that our methods could therefore enable researchers to report unbiased VE estimates even when imperfect tests had to be used. Such methods could also help in the scaling up of TND studies, as tests with limited performance are usually inexpensive and logistically convenient.

Although TND is a relatively new study design, first appearing in a publication in 2005 [27], it has gained broad popularity and is becoming a standard approach in VE studies. One of the largest factors that have contributed to its widespread use is the fact that data collection can be completed within clinical setups [1]. Whereas cohort or case-control studies usually require additional efforts including follow-up or recruitment of non-patients, TND studies only involve patients visiting healthcare facilities with suspects of certain diseases and thus routinely collected clinical data can be easily adapted for analysis. VE studies of influenza, for which TND is most frequently used, often use PCR as a diagnostic tool for better data quality [23]. However, such studies usually involve intensive effort and cost, and thus may only be feasible by large-scale research bodies. Our bias correction methods may open a possibility of wider use of clinical data especially in settings where rapid tests are routinely used for diagnosis. For example, rapid influenza diagnostic tests are routinely used for outpatient clinics and hospitals in Japan, and such clinical data have facilitated a number of TND studies [28-33]. Such studies based on rapid tests could benefit from our methods, as it would provide strong support for the validity of their estimates. Our methods may also be useful in resource-limited settings or for diseases without high-performance diagnostic tools.

Even in resourceful settings where high-performance tests are available, the slight possibility of misclassification might not 

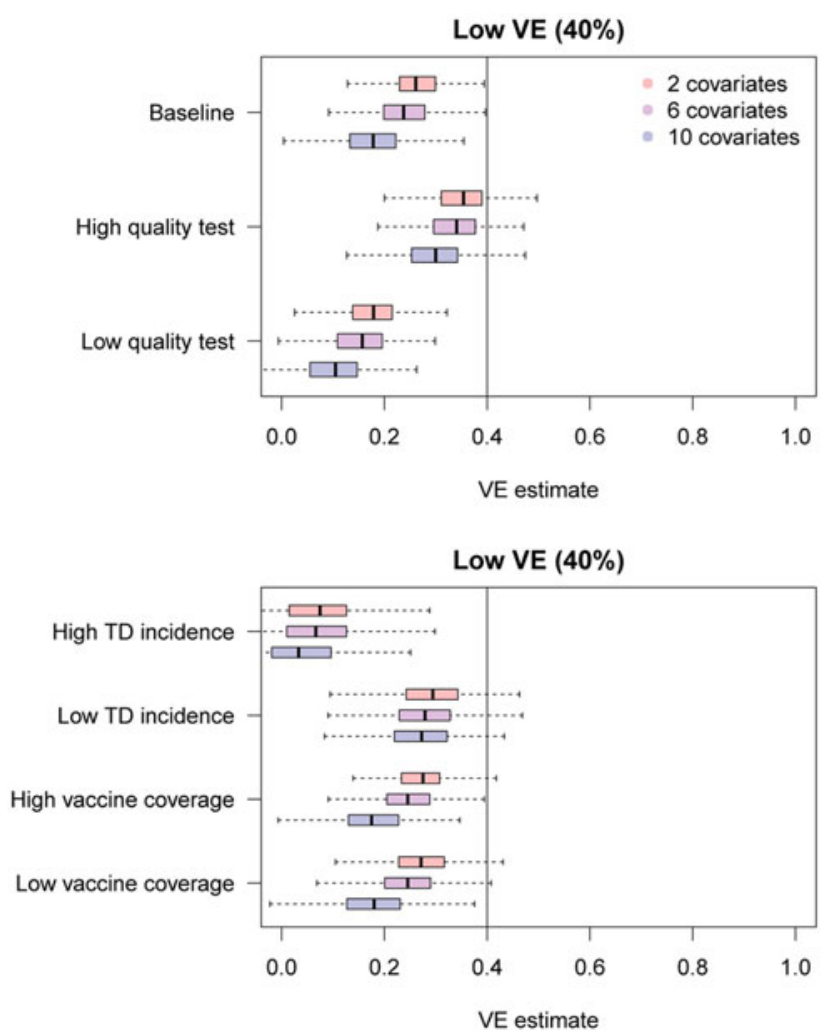
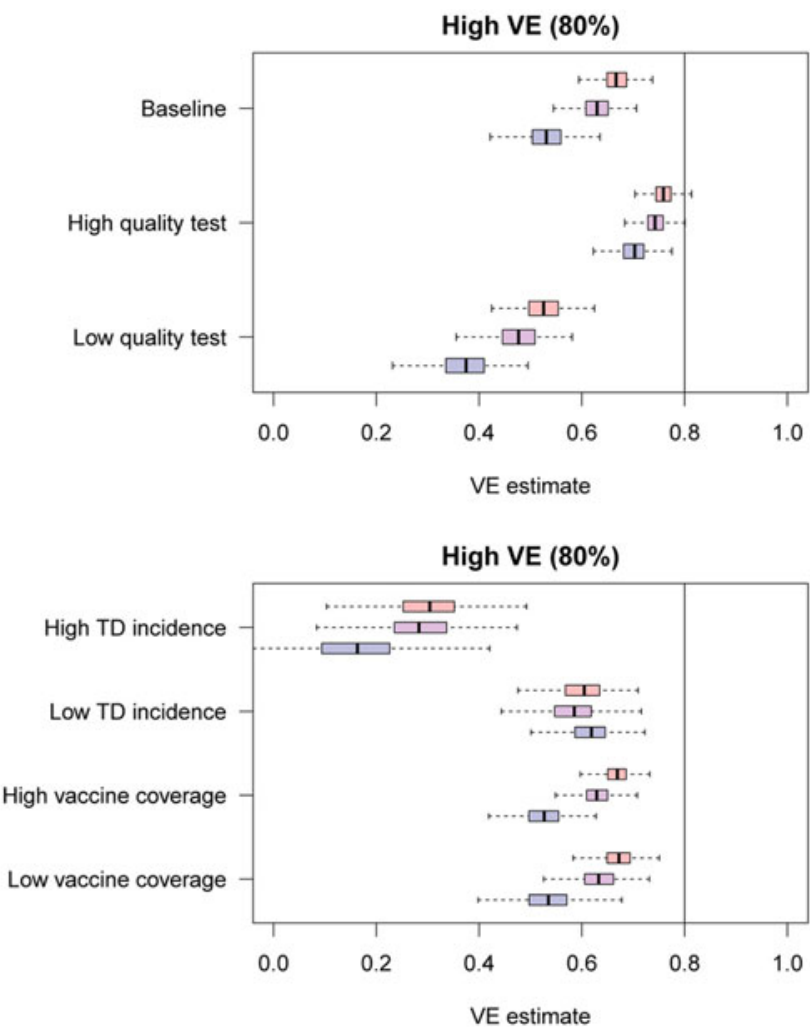

Fig. 7. Bias in raw VE estimates from simulated data in the presence of different numbers of confounding variables. The distributions in red, purple and blue correspond to uncorrected VE estimates in the presence of 2, 6 and 10 confounding variables in addition to the vaccination history.

always be neglected. Although PCR tests are currently used as a gold-standard for influenza diagnosis, their sensitivity and specificity may not be exact $100 \%$; especially, the sensitivity of the test depends not only on microbiological technique but also on the quality of swab samples. In addition, it is suggested that the sensitivity of PCR tests may change during the time course of infection [34] and be sufficiently high only during a limited time window. Our simulation study also indicated that a high heterogeneity in individual characteristics in the study population might increase the bias. Our methods could enable researchers to implement sensitivity analysis by assuming the possible test sensitivity and specificity in such cases.

Our bias correction methods are also intended to be reasonably straightforward for researchers to introduce. Existing estimation tools including software libraries and packages are often used in epidemiological analyses. Incorporating the MO approach, our parametric bootstrapping bias correction method only involves data manipulation and does not require modification of the estimation algorithm. Once multiple sets of data are randomly generated, any type of analysis can be performed as long as the results can be summarised over the MO datasets. Of particular note is that our methods for multivariate analysis allow stratification of sensitivity and specificity among individuals. Therefore, the users can employ more complex misclassification mechanisms including time-varying test performance or test performance affected by individual characteristics. Datasets with a mixture of different diagnostic tools $[3,35]$ can also be handled by applying different values for each test.

There are some limitations to our study. We only focused on misclassification of diagnosis (i.e. misclassified outcomes) and did not consider misclassification of covariates (e.g. vaccine history and other confounding variables), which is another important type of misclassification in TND studies [10]. Further, it is generally not easy to plausibly estimate the sensitivity and specificity for measurement of covariates (e.g. recall bias), which must be known or assumed to implement bias correction. However, if reliable estimates are available, an extension of our approach may yield bias-corrected VE estimates in the presence of covariate misclassification. Moreover, to keep our focus only on diagnostic misclassification, our methods rested on the assumption that other sources of bias in TND studies are non-existent or properly addressed. Potential sources of bias in TND studies have been discussed elsewhere [16, 36, 37], and the researchers conducting TND studies need to carefully consider the possibility of such biases in addition to the diagnostic misclassification. Lastly, it must be noted that our methods depend on the assumed test sensitivity and specificity, and that misspecifying those values can result in an improper correction. The sensitivity and specificity of tests are usually reported by manufacturers in a comparison of the test results with gold-standard tests; however, when such gold-standard tests themselves are not fully reliable or when no available test has satisfactory performance to be regarded as goldstandard, specifying sensitivity and specificity of a test is in principle impossible. Further, test performances reported by manufacturers might lack sufficient sample size or might not be identical to those in the actual study settings. Use of composite reference standards $[38,39]$ or external/internal validation approaches [40] may help overcome these problems.

Although the presence of imperfect diagnosis limits the quality of clinical data, such data can still hold useful information, and 
this information can be transformed into useful insights by appropriate statistical processing. Our bias correction methods were developed primarily for TND studies, but a similar approach could be applied to broader classes of estimation problems with misclassification. Potential areas for future analysis include extension to test data involving continuous quantitative measurements, and coupling with dynamic transmission models. The value of routinely collected data in healthcare settings has become widely recognised with the advancement of data infrastructure, and we believe our methods could help support the effective use of such data.

\section{Conclusion}

Bias correction methods for the TND studies were developed to address potential misclassification bias due to imperfect tests.

Supplementary material. The supplementary material for this article can be found at https://doi.org/10.1017/S0950268820002058.

Acknowledgement. We thank Sheena G. Sullivan for helpful feedback on our early work.

Financial support. This work was supported by the Wellcome Trust (S.F., grant number 210758), (A.J.K., grant number 206250); The Nakajima Foundation (A.E.); the Alan Turing Institue (A.E.); and Lnest Grant Taisho Pharmaceutical Award (A.E.).

Conflict of interest. AE received funding from Taisho Pharmaceutical Co., Ltd.

Data availability statements. This study did not involve original data collection and replication codes are available on GitHub under the MIT License (https://github.com/akira-endo/TND-biascorrection/).

\section{References}

1. De Serres G et al. (2013) The test-negative design: validity, accuracy and precision of vaccine efficacy estimates compared to the gold standard of randomised placebo-controlled clinical trials. Eurosurveillance.

2. Fukushima W and Hirota Y (2017) Basic principles of test-negative design in evaluating influenza vaccine effectiveness. Vaccine.

3. Suzuki M et al. (2017) Serotype-specific effectiveness of 23-valent pneumococcal polysaccharide vaccine against pneumococcal pneumonia in adults aged 65 years or older: a multicentre, prospective, test-negative design study. The Lancet Infectious Diseases 17, 313-321.

4. Prato R et al. (2018) Effectiveness of the 13-valent pneumococcal conjugate vaccine against adult pneumonia in Italy: a case-control study in a 2-year prospective cohort. BMJ Open 8.

5. Araki K et al. (2018) Effectiveness of monovalent and pentavalent rotavirus vaccines in Japanese children. Vaccine 36, 5187-5193.

6. Lopez AL et al. (2018) Effectiveness of monovalent rotavirus vaccine in the Philippines. Scientific Reports 8, 14291

7. Muhsen K et al. (2018) Effectiveness of rotavirus pentavalent vaccine under a universal immunization programme in Israel, 2011-2015: a case control study. Clinical Microbiology and Infection 24, 53-59.

8. Orenstein EW et al. (2007) Methodologic issues regarding the use of three observational study designs to assess influenza vaccine effectiveness. International Journal of Epidemiology.

9. Jackson ML and Rothman KJ (2015) Effects of imperfect test sensitivity and specificity on observational studies of influenza vaccine effectiveness. Vaccine.

10. De Smedt T et al. (2018) Bias due to differential and non-differential disease - and exposure misclassification in studies of vaccine effectiveness. PLOS ONE.
11. Haber M et al. (2018) A comparison of the test-negative and traditional case-control study designs with respect to the bias of estimates of rotavirus vaccine effectiveness. Vaccine 36, 5071-5076.

12 Greenland S (1996) Basic methods for sensitivity analysis of biases.

13. Kleinbaum DG, Kupper LL and Morgenstern H (1982) Epidemiologic Research: Principles and Quantitative Methods. John Wiley \& Sons.

14. Jackson ML and Nelson JC (2013) The test-negative design for estimating influenza vaccine effectiveness. Vaccine 31, 2165-2168.

15. Blackwell M, Honaker J and King G (2017) A unified approach to measurement error and missing data: overview and applications. Sociological Methods and Research.

16. Haber M et al. (2015) A probability model for evaluating the bias and precision of influenza vaccine effectiveness estimates from case-control studies. Epidemiology and Infection 143, 1417-1426.

17. Cowling BJ and Nishiura $\mathbf{H}$ (2012) Virus interference and estimates of influenza vaccine effectiveness from test-negative studies. Epidemiology (Cambridge, Mass.).

18. Cowling BJ et al. (2012) Increased risk of noninfluenza respiratory virus infections associated with receipt of inactivated influenza vaccine. Clinical Infectious Diseases.

19. Sundaram ME et al. (2013) Influenza vaccination is not associated with detection of noninfluenza respiratory viruses in seasonal studies of influenza vaccine effectiveness. Clinical Infectious Diseases.

20. Suzuki M, Camacho A and Ariyoshi K (2014) Potential effect of virus interference on influenza vaccine effectiveness estimates in test-negative designs. Epidemiology and Infection.

21. Leung VK et al. (2016) Concordance of interim and final estimates of influenza vaccine effectiveness: a systematic review. Eurosurveillance.

22. Young B et al. (2018) Duration of influenza vaccine effectiveness: a systematic review, meta-analysis, and meta-regression of test-negative design case-control studies. Journal of Infectious Diseases.

23. Darvishian M et al. (2014) Effectiveness of seasonal influenza vaccine in community-dwelling elderly people: a meta-analysis of test-negative design case-control studies. The Lancet Infectious Diseases.

24. Sidney Burrus C (2012) Iterative reweighted least squares.

25. Rubin DB (2004) Multiple Imputation for Nonresponse in Surveys. Wiley Classics Library. Wiley.

26. Magder LS and Hughes JP (1997) Logistic regression when the outcome is measured with uncertainty. American Journal of Epidemiology 146, 195-203.

27. Skowronski DM et al. (2005) Effectiveness of vaccine against medical consultation due to laboratory-confirmed influenza: results from a sentinel physician pilot project in British Columbia, 2004-2005. Canada Communicable Disease Report.

28. Seki Y et al. (2016) [Effectiveness of influenza vaccine in adults using A test-negative, case-control design -2013/2014 and 2014/2015 seasons-] (Japanese). Kansenshogaku zasshi. The Journal of the Japanese Association for Infectious Diseases.

29. Seki Y, Onose A and Sugaya N (2017) Influenza vaccine effectiveness in adults based on the rapid influenza diagnostic test results, during the 2015/16 season. Journal of Infection and Chemotherapy.

30. Saito N et al. (2017) Negative impact of prior influenza vaccination on current influenza vaccination among people infected and not infected in prior season: a test-negative case-control study in Japan. Vaccine.

31. Shinjoh $\mathbf{M}$ et al. (2018) Inactivated influenza vaccine effectiveness and an analysis of repeated vaccination for children during the 2016/17 season. Vaccine.

32. Ando S (2018) Effectiveness of quadrivalent influenza vaccine based on the test-negative control study in children during the 2016-2017 season. Journal of Infection and Chemotherapy.

33. Sugaya $\mathbf{N}$ et al. (2018) Three-season effectiveness of inactivated influenza vaccine in preventing influenza illness and hospitalization in children in Japan, 2013-2016. Vaccine.

34. Sullivan SG, Feng S and Cowling BJ (2014) Potential of the test-negative design for measuring influenza vaccine effectiveness: a systematic review. Expert Review of Vaccines. 
35. Franke MF et al. (2017) Comparison of two control groups for estimation of oral cholera vaccine effectiveness using a case-control study design. Vaccine.

36. Sullivan SG, Tchetgen EJ and Cowling BJ (2016) Theoretical basis of the test-negative study design for assessment of influenza vaccine effectiveness. American Journal of Epidemiology 184, 345-353.

37. Lewnard JA et al. (2018) Measurement of vaccine direct effects under the test-negative design. American Journal of Epidemiology.

38. Limmathurotsakul D et al. (2012) Fool's gold: why imperfect reference tests are undermining the evaluation of novel diagnostics: a reevaluation of 5 diagnostic tests for leptospirosis. Clinical Infectious Diseases.

39. Naaktgeboren CA et al. (2013) Value of composite reference standards in diagnostic research. BMJ (Clinical research ed.).

40. Kahn SR et al. (2018) Comparing external and internal validation methods in correcting outcome misclassification bias in logistic regression: a simulation study and application to the case of postsurgical venous thromboembolism following total hip and knee arthroplasty. Pharmacoepidemiology and Drug Safety.

\section{Appendix}

Mathematical notations in section 'Methods and results'

\section{Characterising bias in TND studies}

$r_{1}, r_{0}:$

$n_{V}, n_{U}$ :

$\gamma$ :

$m, m_{U}$

$\mu:$ prevalence of TD/ND in the unvaccinated population vaccinated and unvaccinated population size the relative risk of TD in the vaccinated population relative to the unvaccinated $(\mathrm{VE}=1-\gamma)$ the probability of medical attendance given ND in vaccinated/unvaccinated population factor for the probability of medical attendance given TD in relative to that given ND
$x_{V}, x_{U}, x_{+V}, x_{+U}, x_{-V}, x_{-U}$ $y_{V}, y_{U}, y_{+V}, y_{+U}, y_{-V}, y_{-U}$ $X_{V}, X_{U}, Y_{V}, Y_{U}$ :

$q:$ $\alpha, \beta$ :

$C:$

$c:$

\section{Bias correction in univariate analysis}

$\gamma^{*}$ : $\quad$ corrected odds ratio

$\sigma^{*}$ : $\quad$ shape parameter of the log-normal distribution that gives the confidence interval of $\gamma^{*}$ (see Equation (4))

$\lambda_{V}, \lambda_{U}$ : 'baseline medical attendance', the mean number of vaccinated/ unvaccinated patients when vaccine has no effect $(\mathrm{VE}=0)$

\section{Bias correction in multivariate analysis}

$\xi=\left(\xi^{1}, \xi^{2}, \ldots, \xi^{n}\right)$ : covariates included in the model, where $x i^{1}$ denotes vaccination history

$Z_{i}, z_{i}$ :

$p_{1}(\xi), p_{0}(\xi)$

$\theta, \theta^{*}:$

$\pi_{Z_{i}}\left(\xi_{i}\right)$ :

$\tilde{z}_{i}^{j}$ :

$\tilde{\phi}_{i+}, \tilde{\phi}_{i-}:$ observed test result and true disease state for individual $i$ probability that the true test result is positive/negative for an individual with covariates $\xi$ model parameter for $p_{1}(\xi)$ and its estimate probability for the observed test result $Z_{i}$ imputed disease state of individual $i$ in the $j$-th imputed dataset $\tilde{D}^{j}$

incorrect

$\varepsilon^{j}: \quad \quad$ point estimate for VE from the $j$-th imputed dataset $\tilde{D}^{j}$ 